\title{
Una propuesta de análisis cualitativo de políticas públicas desde un enfoque cognitivo
}

\author{
A proposal for the qualitative analysis of public policies from a cognitive approach \\ Pablo Gustavo Rodriguez \\ Universidad Nacional de La Plata- \\ Facultad de Ciencias Naturales y Museo, Argentina \\ pablogrod@fcnym.unlp.edu.ar
}

\section{ReSUMEN:}

Se presenta el procedimiento de análisis desarrollado en el curso de una investigación sobre el concepto del desarrollo en los programas sociales bonaerenses. Se expone, como contexto, el diseño general de la mencionada investigación, incluyendo brevemente sin coma objetivos, unidades de análisis, características del corpus y marco teórico-metodológico, para luego describir en detalle los procedimientos analíticos en tres momentos: reducción de datos, construcción de conceptos teóricos mediante la agregación de códigos y comparación de hallazgos a través de las unidades de análisis. Se sugiere que el enfoque y el procedimiento descritos pueden ser de utilidad en otros estudios de políticas públicas.

Palabras clave: Análisis cualitativo, Análisis de discurso, Codificación, Políticas públicas, Enfoque cognitivo.

\begin{abstract}
:
The procedure of analysis developed in the course of research on the concept of development in Buenos Aires social programs is presented. The general design of the aforementioned research is presented briefly as a context, including objectives, units of analysis, corpus characteristics and theoretical-methodological framework. Following a detailed description of the analytical procedures are given in its three moments: data reduction, construction of theoretical concepts through the aggregation of codes and comparison of findings through the units of analysis. It is suggested that the approach and procedure described above may be useful in other public policy studies.
\end{abstract}

KEYWORDs: Qualitative analysis, Discourse analysis, Coding, Public policy, Cognitive approach.

\section{INTRODUCCIÓN}

El procedimiento analítico que se expone en el presente artículo fue desarrollado en la tesis doctoral "Las concepciones del desarrollo en los programas de promoción de emprendimientos productivos del Ministerio de Desarrollo Humano de la Provincia de Buenos Aires (1990-2007)" (Rodríguez, 2013), realizada con el apoyo de una Beca de Formación Superior de la Universidad Nacional de La Plata entre 2008 y 2010, bajo la dirección de la Dra. Amalia Eguía. El concepto aludido no es realmente nuevo pero sí la manera de definirlo, incluyendo su operacionalización para el análisis de políticas públicas desde un enfoque cognitivo.

En dicha investigación fueron analizados documentos de doce programas provinciales de promoción de emprendimientos productivos que funcionaron entre 1989 y 2007, dependientes en forma sucesiva de seis organismos provinciales: Subsecretaría de Acción Social (SAS) del Ministerio de Acción Social (1984-1991), Subsecretaría de Organización Comunitaria (SOC) del Ministerio de Salud y Acción Social (1991-1994), Consejo Provincial de la Mujer (1991-1995), Consejo Provincial de la Familia y Desarrollo Humano (CPFyDH, 1995-2002), Ministerio de Desarrollo Humano y Trabajo (MDHyT, 2002-2004) y Ministerio de Desarrollo Humano (MDH, 2004-2007). Este organismo corresponde al actual Ministerio de Desarrollo Social (MDS).

Los programas considerados fueron: Programa Alimentario Integral y Solidario (PAIS), Programa Provincial de Microempresas (PPM), Manos Bonaerenses (MB), Mujer Isleña (MI), Mujer de campo 
(MC), Programa de Desarrollo Local (PRODEL), Programa Unificado de Estrategias Bonaerenses para la Organización (PUEBLO), Programa Provincial de Economía Social (Banca Social), Programa de Fortalecimiento Socioproductivo para Comunidades Rurales (Volver), Huertas Bonaerenses (HB), Iniciativas para el Desarrollo Local (IDL) y El Trabajo dignifica (ETD).

También se analizaron documentos de tres organismos internacionales que orientaron el diseño de los mencionados programas provinciales mediante consultorías y acciones de capacitación. Estos fueron el Programa de las Naciones Unidas para el Desarrollo (PNUD), el Banco Interamericano de Desarrollo (BID) y los Congresos de Desarrollo Social de las Naciones Unidas.

El estudio se propuso determinar cuáles fueron los cambios y las continuidades en las concepciones acerca del desarrollo en los programas de promoción de emprendimientos productivos impulsados por el MDH entre 1989 y 2007, a nivel del enfoque utilizado y de las prácticas prescritas para la implementación de los programas.

El corpus textual estuvo compuesto por 102 documentos producidos por los programas y organismos provinciales, 41 documentos de los organismos internacionales, y por la transcripción de 41 entrevistas de bajo grado de estructuración administradas a empleados, ex-empleados, funcionarios, ex-funcionarios de los organismos provinciales y ex-consultores de los organismos internacionales que intervinieron en los organismos provinciales considerados. Todos estos textos fueron digitalizados a fin de poder someterlos al análisis de discurso asistido por computadora con apoyo del software QSR-NVivo . ${ }^{1}$

La estrategia metodológica general fue de tipo cualitativa, etnográfica, puesto que las preguntas de investigación refieren a cuestiones conceptuales, de sentido, para las cuales dicha estrategia resulta ser la más adecuada. En esta decisión influyó una concepción de las aproximaciones cualitativas como la que expresan Vasilachis de Gialdino (1992) y Glaser y Strauss (1967), según la cual este tipo de estudios no persigue la verificación o refutación de una hipótesis derivada de una teoría preexistente, sino la explicación del fenómeno estudiado mediante la propuesta de un modelo teórico como producto final del proceso investigativo. Las principales técnicas utilizadas fueron la observación participante, la realización y análisis de entrevistas y la recopilación y análisis de documentos.

La noción de desarrollo fue considerada como una categoría nativa o emic (Pike, 1954) usada por los actores sociales (personas e instituciones implicadas) en su vida cotidiana. El análisis buscó identificar el sentido de las diferentes modalizaciones que recibe este concepto en el uso que hacen de él los actores sociales (Magariños de Morentín et al, 1990), y no como una categoría de las ciencias sociales. Por ese motivo el marco teórico no incluyó una definición de desarrollo.

Un principio que orientó el estudio fue el de identificar la fuente de las concepciones de desarrollo que adoptaron los programas sin pre-suponer la existencia de conexiones o influencias que no se pudieran documentar. Para ello se siguió la pista del intertexto ${ }^{2}$ (la referencia más o menos explícita en las fuentes a un texto base), como recomienda la semiótica de enunciados (Magariños de Morentín et al., 1990, 1996). Ello condujo a documentos de algunos organismos internacionales, como el Banco Interamericano de Desarrollo (BID) y el Programa de las Naciones Unidas para el Desarrollo (PNUD).

En todo momento se evitó proceder sobre la base del supuesto de que por su solo alcance internacional las posiciones de estos organismos tuvieron una incidencia efectiva en la determinación de las políticas que siguen los organismos provinciales. Cuando se establecieron conexiones entre ambos tipos de organismos fue sobre la base de la existencia documentada de una incidencia efectiva cuya naturaleza y alcances pueden ser descritos en vez de conjeturados.

El PNUD y el BID son mencionados en los documentos de los programas provinciales como autores de enfoques asumidos como propios por los organismos provinciales. Por otra parte, la información relevada muestra que aquellos además trabajaron activamente para inducir la adopción de dichos enfoques por parte de los organismos provinciales, a través de consultorías y capacitaciones en las que les transfirieron un conjunto de saberes técnicos diseñados especialmente para su implementación práctica. 
El marco teórico-metodológico articuló de una forma original cuatro vertientes:

a) Un enfoque cognitivo de las políticas públicas que las entiende como esquemas complejos y negociados por múltiples actores, consistentes en valores, estrategias, formas de acción e instrumentos que buscan responder a cuestiones aceptadas como problemas sociales. Este enfoque se inspira en trabajos de Oszlak y O'Donnell (1995), Aguilar Villanueva (1996), Aguilar Villanueva et al (1993), Murray Edelman (1991), Pierre Bourdieu (1997), Paul Sabatier (1998) y Antonio Camou (1997), sobre las políticas públicas y en los estudios de antropología cognitiva sobre esquemas culturales (Rice, 1980, Casson, 1983, entre otros).

b) Un enfoque inspirado en la obra tardía de Michel Foucault $(2007,2008)$ que permite entender dichos esquemas como una forma de gobierno de los otros "a distancia", a través de sí mismos, de origen neoliberal. Este enfoque aporta una mirada histórica y social sobre la construcción de la subjetividad, y contribuye con los conceptos de gubernamentalidad, dispositivo, tecnologías del yo y régimen de verdad. Esta línea de pensamiento incluye trabajos más recientes de autores como Rose (1992, 1996, 2003, 2007), Rose y Miller (1992), Miller y Rose (1988, 1990), Rose, O’Malley y Valverde (2006), O’Malley y Palmer (1996).

De modo similar a como Foucault estudió las prácticas para el tratamiento de los enfermos, los locos, los criminales, etc., con las que se pretendía convertirlos en sujetos "normales", se pueden describir las prácticas de promoción del desarrollo como una tecnología para convertir a los pobres y desocupados en un tipo particular de sujeto, gubernamentalizable a partir de su libertad.

c) Un enfoque crítico de las relaciones entre cognición, discurso y sociedad, tomado de la obra de Teun van Dijk (1996a, 1996b, 2000), que permite acceder a la cognición social a través del discurso. Este enfoque contribuye con una particular noción de ideología entendida como sistemas de creencias fácticas y evaluativas que conforman esquemas o modelos mentales residentes en la memoria semántica o social, representables como redes conceptuales que expresarían conexiones neuronales. Las creencias pueden representarse en forma simplificada como redes de proposiciones (o enunciados). En tanto los conjuntos de creencias, como las ideologías, se representan como diferentes tipos de esquemas (guiones, marcos, escenarios, etc.).

Entre las creencias que integran las ideologías se encuentran también las actitudes y los valores. Las primeras son creencias evaluativas generales compartidas socialmente. Los segundos representan objetivos ideales por los cuales luchar, reconocidos culturalmente, que fundan un orden moral.

Mientras que los modelos mentales son de carácter personal y comportan las representaciones e interpretaciones subjetivas de acontecimientos derivados de la experiencia individual, los esquemas descritos anteriormente son de carácter social o cultural y han sido objeto de estudio de la antropología cognitiva. Los modelos mentales son la interfaz entre las representaciones sociales compartidas y las prácticas personales.

Me propuse entonces identificar los esquemas culturales para el concepto de "desarrollo" entre los trabajadores y funcionarios del Ministerio de Desarrollo Humano. Dichos esquemas constituirían la memoria institucional ${ }^{3}$ del organismo entendida, por analogía con la memoria semántica, como el conjunto de tales esquemas que posee una institución para definir y tratar los problemas que son de su competencia, y de los cuales cada integrante del grupo tiene sus propia versión personal o modelo mental. Dadas las relaciones que establece van Dijk entre sociedad, cognición y discurso, el análisis de este último se constituye en una vía factible de acceso a las estructuras cognitivas compartidas por el grupo.

d) El enfoque adoptado se completa con una teoría propuesta para explicar las crisis del sistema capitalista, que permite entender cómo se articulan las estructuras cognitivas, sociales y económicas para "asegurar la compatibilidad de los comportamientos económicos descentralizados de las unidades de producción y de los individuos sin que dichos agentes se hayan puesto voluntariamente de acuerdo entre sí" (Neffa, 2008, 217).

Básicamente, la teoría de la regulación o regulacionismo explica la crisis del fordismo y, con ella, el marco histórico en el que surgen los programas analizados, que es el de la reforma de los Estados argentino y bonaerense. Se consideraron sobre todo trabajos de Sergio Boisier (2005), Alain Lipietz (1988, 1994), Benko y Lipietz (1994) y Lipietz y Leborgne (1994). 


\section{EL PROCEDIMIENTO DE ANÁLISIS}

Como se dijo anteriormente, el análisis fue realizado con la ayuda del software Nvivo. Tras cargar todos los textos en el mismo se procedió a crear nodos de caso para cada unidad de análisis, considerando fundamentalmente dos tipos de tales unidades: programas y organismos.

Cada documento fue asignado al nodo de caso correspondiente. Se definió el conjunto de atributos (variables demográficas) que caracterizarían cada tipo de unidad de análisis (caso) y sobre la base de ello se definió una "hoja de clasificación" para cada una, vale decir, una matriz de datos, de casos por variables.

Para los programas se estableció como único atributo "gestión", entendiendo por "gestión" los elencos de funcionarios políticos a cargo del organismo, que incluyen desde el Ministro o Secretario de Estado hasta los directores de línea. Las gestiones en este estudio fueron la Gestión Duhalde, Gestión Ruckauf, Gestión West y MDH. ${ }^{4}$ En el caso de los organismos se consideró sólo su "dependencia" como provincial o internacional.

Tras estos pasos preparatorios se procedió a la lectura secuencial de cada documento identificando la presencia de temas relevantes y creando los códigos (o nodos) correspondientes. Ello incluye tres tipos de categorías: tanto categorías emic como categorías etic de dos clases:

- Categorias "bottom-up": creadas a partir de conceptos efectivamente presentes en las fuentes (Pike, 1954; Richards y Richards, 1995). Se reconocen dos subtipos:

- Categorías emic o nativas, ya comentadas.

- Categorías emergentes creadas por el analista pero sugeridas por la lectura de las fuentes).

- Categorías etic o top-down: tomadas de la bibliografía teórica.

El procedimiento básico de codificación se realizó como sugieren Glaser y Strauss (1967): generación de categorías mediante comparación constante, definición, delimitación y aplicación sistemática hasta alcanzar el punto de saturación teórica y posterior articulación en un modelo conceptual.

En un primer momento el análisis se orientó a la identificación de todas las modalizaciones de "desarrollo" presentes en el corpus, para las cuales se crearon los códigos correspondientes.

Se adoptó el concepto de modalización que utiliza la semiótica de enunciados. Esta metodología asume que "todo lo efectivamente dicho se corresponde con una posibilidad de decir lo preexistente" (Magariños de Morentín, 1998), posibilidad que no es individual, sino que el productor del texto en cuestión comparte con la comunidad a la que pertenece. Vale decir que un enunciado es una actualización de una relación semántica entre términos que estaba disponible para el enunciador antes de verbalizarla. Esta posibilidad cognitiva es lo que representa la modalización. Cualquier adjetivación, complemento o cláusula subordinada son modalizadores.

Las principales modalizaciones halladas fueron las de desarrollo comunitario, desarrollo social, desarrollo humano, desarrollo local, desarrollo económico y desarrollo socioproductivo. Esta búsqueda estuvo guiada por las preguntas ¿qué clases de desarrollo existen? y ¿qué es el desarrollo?

Asimismo, se buscó responder la pregunta ¿cómo se alcanza cada uno de los tipos de desarrollo mencionados? y se crearon los códigos respectivos. ¿Qué aspecto tienen las indicaciones sobre la manera de alcanzar el desarrollo? En varios documentos analizados se expresan con cierta claridad algunos principios rectores o lineamientos estratégicos para sus prácticas. Como ilustración, se reproduce a continuación un pasaje correspondiente al programa Volver debido a que es tal vez el que los expone con mayor claridad:

"Este tipo de intervención genera las condiciones para concretar un modelo de gestión social diferente con estas características:

- Integralidad

- Inclusión social 
- Desarrollo local con equidad

- Gestión asociada

- Fortalecimiento de las capacidades locales

- Descentralización

- Complementariedad

- Respeto por la identidad cultural y tradiciones

- Recuperación de los saberes populares

- Mejoramiento de la calidad de vida del hábitat desde la lógica de Desarrollo Humano

- Planificación

- Cuidado del medio ambiente

- Sustentabilidad

- Interrelación con otras comunidades

- Generación de trabajo genuino

- Expansión de ciudadanía." (Documento: Volver- Propuesta institucional, p. 4).

Otros programas presentan entre sus principios rectores: asociativismo, equidad, autonomía y autogestión de los destinatarios, flexibilidad, participación, organización comunitaria, asistencia, promoción, empoderamiento, focalización, solidaridad, etc.

El conjunto de estos principios caracteriza lo que los documentos del CPFyDH denominan el modelo de gerenciamiento social, o como lo llamaron algunos entrevistados, un modelo de intervención. La estrategia seguida en el análisis de los discursos relevados, inspirada en los trabajos de T. van Dijk, se orientó precisamente a identificar y caracterizar dichos modelos de intervención entendidos como modelos mentales sobre qué es el desarrollo y cómo debe ser promovido. Tales modelos están conformados por los principios orientadores de las prácticas que mencionan los documentos de los programas y organismos así como los entrevistados.

En un segundo momento, ante la diversidad y heterogeneidad de dichos principios se los clasificó en cinco tipos (categorías supraordinadas surgidas a partir de la integración de las anteriormente identificadas), que fueron denominados: objetivos estratégicos, estrategias de intervención, tecnologías de gestión, instrumentos y objetos de intervención. La Figura 1 muestra un esquema conceptual de las relaciones entre los diferentes tipos de componentes de los modelos de intervención. 
FIGURA 1

Componentes de los modelos de intervención

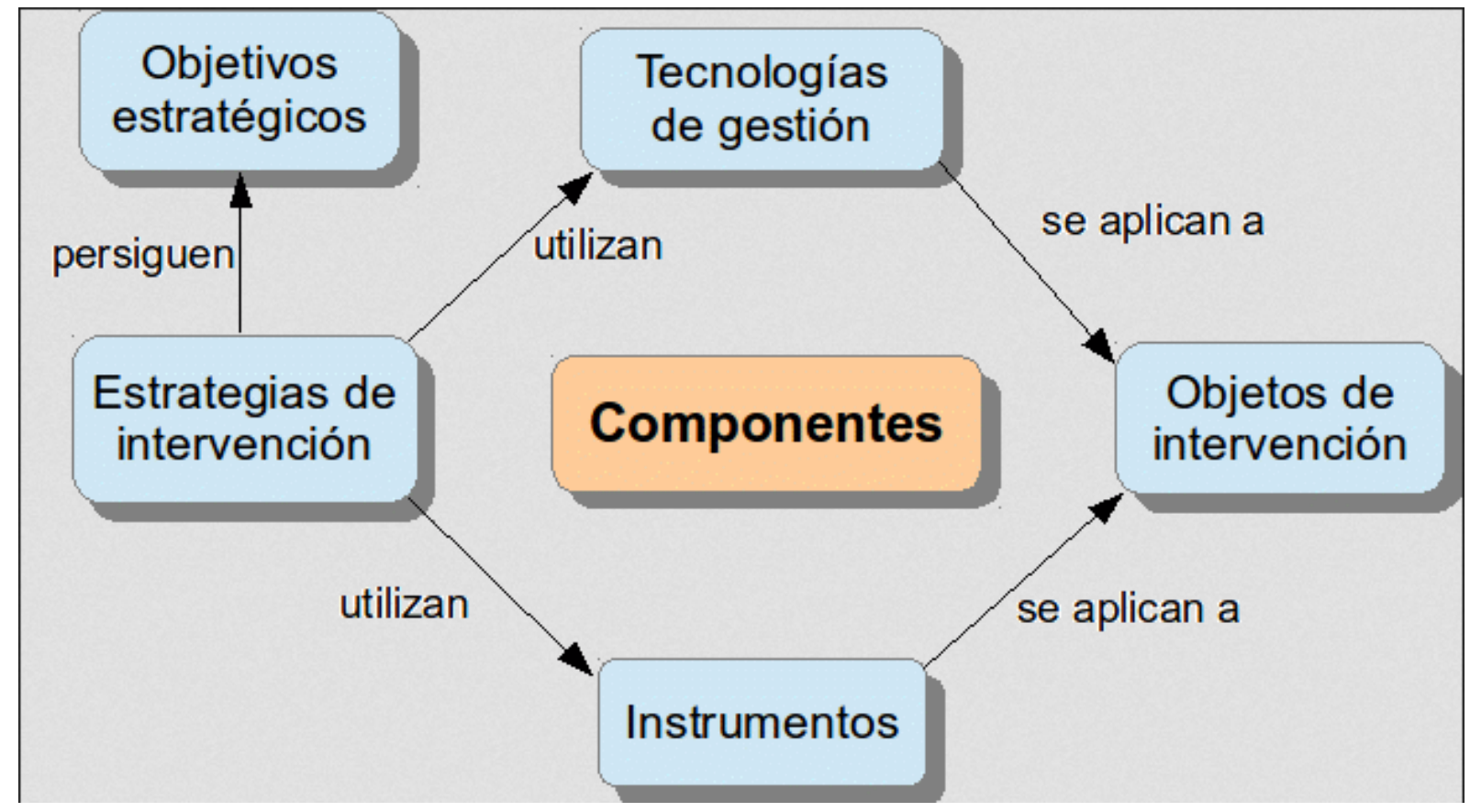

Los objetivos estratégicos (OE) son fines en sí mismos, estados finales o condiciones que se pretenden alcanzar por ser considerados valiosos, en el sentido de ser un valor público, como la equidad, la inclusión, la integración social, la eficiencia, la autonomía o autogestión, la sinergia o la sustentabilidad. En el sentido otorgado aquí, los objetivos estratégicos se aproximan a lo que Rose $(2003,223)$ denomina "teleologías".

Las estrategias de intervención (EI) son modos de abordaje o formas de perseguir los objetivos estratégicos. Son los criterios generales, los grandes lineamientos o los principios metodológicos que orientan la acción, por oposición a las operaciones, para regular los comportamientos de las personas de conformidad con los OE. Representan los principios de diseño de los programas analizados. Son líneas de acción prescritas en forma explícita, aunque nada garantiza que las prácticas efectivamente observadas se guíen por ellas. Algunas estrategias halladas fueron: integralidad, solidaridad, protagonismo, asociacionismo, autoempleo, empleabilidad, participación, flexibilidad, fortalecimiento del tejido social o de la sociedad civil y promoción de la organización comunitaria.

Las tecnologías de gestión (TG) son conjuntos de conocimientos que, como un saber-hacer especializado, se aplican a la realización de una serie de operaciones sistemáticas. Algunos de ellos provienen del campo profesional de la administración de empresas, como la planificación participativa y la estratégica, la externalización o tercerización (outsourcing), la focalización (targeting o segmentación de mercados), el tablero de comando y el FODA. También se consideraron tecnologías de este tipo a la gestión asociada, la gestión por resultados y la descentralización.

La noción de tecnologías de gestión es de uso corriente en las ciencias de la administración (Bustos Coral, 2007; Rigby, 2011) y la acepción que recibe en este trabajo es muy cercana, con la diferencia de que aquí son consideradas tecnologías en el sentido que Foucault (2008) les da a las "tecnologías del yo", vale decir, como componentes de un dispositivo de gubernamentalidad.

Los instrumentos de intervención (o simplemente "instrumentos") son artefactos más concretos que constituyen un medio para alcanzar los objetivos estratégicos. Pueden ser

- formas organizativas, como los Consejos Locales Económico Sociales (CLES), las Unidades Técnicas Locales (UTL) o los comités de crédito; 
- materiales de uso administrativo, como los formularios de solicitud y los registros de beneficiarios, o

- productos de los programas, como los microcréditos, los subsidios, la capacitación o la asistencia técnica.

La mayoría de ellos se aplican en correspondencia con alguna tecnología específica. Por ejemplo, los CLES son instrumentos de gestión asociada, un taller puede ser un instrumento de planificación participativa y un fondo rotatorio, un instrumento de acceso al crédito.

Finalmente, los objetos de intervención (OI) son aquellos fenómenos, dimensiones o aspectos de la realidad social que se pretende modificar con la intervención. En el estudio referido, algunos de estos objetos son la desocupación, la pobreza, la vulnerabilidad, la informalidad, el riesgo social, la calidad de vida, las condiciones de vida, el territorio, el despoblamiento, las necesidades de la población objetivo, etc.

En este trabajo se entiende un modelo de intervención como un dispositivo de gubernamentalidad en sentido foucaultiano compuesto por una combinación particular de principios orientadores y de prácticas prescritas de los programas sociales, entre los que se pueden reconocer objetivos estratégicos, estrategias de intervención, tecnologías de gestión, instrumentos y objetos de intervención. ${ }^{5}$

El tercer momento del análisis consistió en identificar los modelos de intervención presentes en cada organismo, gestión y programa considerado a partir del análisis de sus documentos, y en cada grupo de entrevistados (funcionarios y trabajadores), para luego compararlos con el fin de comprobar sus semejanzas y diferencias, y su cambio o continuidad a través de las distintas gestiones. ${ }^{6}$

La comparación se realizó considerando, por una parte, la presencia o ausencia de los diferentes componentes de los modelos de intervención en cada grupo de fuentes así como su peso relativo en términos de la cantidad de texto codificado (medido en cantidad de pasajes, de palabras o de fuentes). Este tipo de comparación se realizó con la ayuda de tablas de frecuencias y porcentajes y con algunos gráficos de barras cuyo valor es apenas descriptivo, ya que los datos producidos no son aptos para ser procesados con técnicas de estadística inferencial, debido a que el corpus textual no constituye una muestra estadísticamente representativa.

Por otra parte, se efectuó la comparación del significado otorgado a cada componente por cada grupo de fuentes, mediante diferentes matrices cualitativas (Bazelay, 2009). A este último tipo de comparación se le otorgó mayor importancia y sus resultados se presentaron en forma discursiva.

Las Tablas 1 a 5, al final de este artículo, muestran los principales objetivos, estrategias, tecnologías, instrumentos y objetos que se identificaron en el corpus.

\section{CONCLUSIONES}

No es habitual hallarse con una exposición detallada de los procedimientos analíticos seguidos en una investigación. La práctica habitual en las publicaciones es la de exponer el marco teórico y los resultados y, en el mejor de los casos, apenas mencionar los métodos o técnicas de análisis utilizados. Desde luego que las limitaciones de extensión que las publicaciones periódicas imponen a los artículos no permiten una exposición detallada de toda una investigación. Por ello, la publicación de artículos especialmente dedicados a la exposición de los procedimientos de análisis es muy deseable.

En este trabajo se ha expuesto en detalle el procedimiento de codificación y la construcción de un modelo conceptual, seguido en un estudio cualitativo de un conjunto de documentos y entrevistas referidos a un grupo de programas de desarrollo de la provincia de Buenos Aires.

En síntesis. dicho análisis reconoce tres momentos: uno de reducción de datos mediante la construcción de categorías analíticas y la codificación de las fuentes, como sugieren los fundadores de la Grounded Theory; uno de agregación de categorías para crear categorías supraordinadas o conceptos de mayor nivel, y un tercero 
de comparación de los hallazgos a través de las unidades de análisis, con el fin de constatar su distribución en el corpus.

Se ha mostrado que las categorías fueron construidas para responder a ciertas preguntas de investigación, como ¿qué tipos de desarrollo existen? y ¿cómo se alcanzan?

Se constata la importancia que tiene para el análisis el contar con un marco teórico y metodológico, como han sido en este caso algunos conceptos y principios de la semiótica de enunciados y el enfoque cognitivo sobre la ideología representado por la obra de T. van Dijk. Estos marcos permitieron orientar el proceso de generación de conceptos sobre la base de la búsqueda de las diferentes modalizaciones del concepto de desarrollo, así como organizar los primeros códigos en categorías de mayor nivel como la de "modelo de intervención", entendido como un modelo mental sobre el desarrollo.

En lo que hace específicamente al estudio de políticas públicas, se ha propuesto como un instrumento útil para su análisis el mencionado concepto de modelo de intervención. Se lo ha definido operativamente como la articulación coherente de un conjunto de objetivos estratégicos, estrategias de intervención, tecnologías de gestión, instrumentos y objetos de intervención. Y se ha propuesto entender teóricamente tales modelos como dispositivos de gubernamentalidad en sentido foucaultiano, consistentes en una combinación particular de principios orientadores y de prácticas o acciones de los programas sociales, que conforman una tecnología de gobierno orientada a modelar o remodelar los mecanismos de autorregulación de la conducta de los individuos.

Se ha mostrado cómo el uso de los modelos de intervención como instrumento de análisis permitió identificar y caracterizar las nociones de desarrollo presentes en un conjunto de programas sociales bonaerenses, y vincularlas a cada una de las gestiones de dichos organismos y a sus respectivos programas.

Finalmente, se sugiere que la noción de modelo de intervención tal como es presentada en este artículo puede constituir un instrumento fructífero para el análisis de otras políticas públicas diferentes de las referidas en este trabajo.

TABLA 1

Principales Objetivos

Estratégicos

\begin{tabular}{|l|l|}
\hline Ampliación de la base empresarial & Equidad \\
Autonomía-autogestión-sujeto activo & Equilibrio macroeconómico \\
Bienestar-prosperidad & Gobernabilidad - estabilidad \\
Crecimiento económico & Inclusión social \\
Eficacia & Integración social \\
Eficiencia & Responsabilización \\
Empoderamiento & Sostenibilidad \\
\hline
\end{tabular}


TABLA 2

Principales Estrategias de Intervención

\begin{tabular}{|l|ll|}
\hline Flexibilidad & \multicolumn{2}{|l|}{ Económicas: } \\
Fortalecimiento & - & Asociativismo \\
Integralidad & - & Autoabastecimiento \\
Participación & - & Autoempleo \\
Protagonismo & - & Empleabilidad \\
Solidaridad & - & Emprendedorismo \\
Organización Comunitaria & - & Generación de empleo \\
Redes & - & Generación de ingresos \\
Capital social & - Subsistencia \\
\hline
\end{tabular}

TABLA 3

Principales Tecnologías de Gestión

\begin{tabular}{|c|c|}
\hline $\begin{array}{l}\text { Evaluación-seguimiento-monitoreo } \\
\text { Descentralización } \\
\text { Tercerización, externalización } \\
\text { (outsourcing) } \\
\text { Focalización (targeting) } \\
\text { (Nueva Gestión Pública o por } \\
\text { resultados) } \\
\text { (Gerencia(miento) social) }\end{array}$ & $\begin{array}{l}\text { Planificación } \\
\text { - } \quad \text { P. estratégica } \\
\text { - } \quad \text { P. participativa } \\
\text { - } \quad \text { Diagnóstico participativo o comu- } \\
\text { nitario } \\
\text { - } \quad \text { Proyectos } \\
\text { Gestión asociada } \\
\text { - } \quad \text { Articulación de actores } \\
\text { - } \quad \text { Nueva institucionalidad }\end{array}$ \\
\hline
\end{tabular}


TABLA 4

Principales Instrumentos

\begin{tabular}{|l|l|}
\hline Organizativos & Productos \\
\hline CLES & Microcréditos o microfinanzas (Fondos \\
Consejos Consultivos & rotatorios, fodeles) \\
Consejos de Desarrollo de la & Subsidios \\
comunidad & Capacitación \\
Consejos locales de desarrollo & Asistencia técnica \\
Consorcio de Gestión & Ferias de comercialización \\
CTP & Eventos, Jornadas \\
Mesa Local & Rondas de negocios \\
Mesa Distrital & Exención tributaria \\
UTL & Semillas y herramientas \\
Otras formas de Gestión Asociada & \\
\hline
\end{tabular}

TABLA 5

Principales Objetos de Intervención

\begin{tabular}{|l|l|}
\hline Calidad de vida & Informalidad \\
Capital Humano RRHH & Lo local \\
Condiciones de vida & Necesidades básicas \\
Desigualdad & Necesidades sentidas \\
(Des)ocupación desempleo & Nivel de vida \\
(Des)poblamiento & Pobreza \\
Exclusión social & Riesgo social \\
& Territorio \\
& Vulnerabilidad \\
\hline
\end{tabular}

ReFERENCIAS BibLIOgRÁficas

Aguilar Villanueva, L. F. (1996). La hechura de las politicas. México: Miguel Ángel Porrúa.

Aguilar Villanueva, L. F., Elder, C. D., Cobb, R. W., Nelson, B. J., Downs, A. y Rittel, H. W. J. (1993). Problemas Públicos y Agenda de Gobierno. México: Porrúa.

Bazeley, Pat (2009). Analyzing qualitative data: more than «identifying themes». Malaisyan Journal of Qualitative Research, 2, 6-22. 
Benko, G. y Lipietz, A. (1994). Las regiones que ganan. Distritos y redes. Los nuevos paradigmas de la geografía económica. Politica y sociedad. Valencia: Edicions Alfons el Magnanim - Generalitat Valenciana.

Bertranou, J. (2004). Notas sobre el concepto de memoria institucional. En Julián Bertranou, J.; Palacio, M. y Gerardo M. Serrano (Comps.) En el pais de no me acuerdo. (Des)memoria institucional e historia de la política social en la Argentina (pp. 117-143). Buenos Aires: Prometeo libros.

Boisier, S. (2005). ¿Hay espacio para el desarrollo local en la globalización? Revista de la CEPAL, (85), 47-62.

Bourdieu, P. (1993). Cosas dichas. Barcelona: Gedisa. Colección El mamífero parlante (Vol. 1).

Bourdieu, P. (1997 [1991]). Espíritus de Estado. Génesis y estructura del campo burocrático. En Razones prácticas. Sobre la teoría de la acción (pp. 91-138). Barcelona: Anagrama.

Bustos Coral, H. D. (2007, Julio 18). Tecnologías de gestión - TG. GestioPolis. Recuperado de http://www.gestiopol is.com/tecnologia/tecnologias-de-gestion-en-la-era-del-conocimiento.htm.

Camou, A. (1997). Los consejeros del príncipe. Saber técnico y política en los procesos de reforma económica en América Latina. Nueva Sociedad, 152, 54-57.

Casson, R. W. (1983). Schemata in Cognitive Anthropology. Annual Review of Anthropology, 12, 429-462.

Charaudeau, P. y Maingueneau, D. (2005). Diccionario de análisis del discurso. Buenos Aires: Amorrortu.

Edelman, M. J. (1991). La construcción del espectáculo político. Buenos Aires: Manantial.

Foucault, M. (2007). Nacimiento de la biopolitica: curso en el Collège de France (1978-1979). Buenos Aires: FCE.

Foucault, M. (2008). Tecnologias del yo y otros textos afines. Pensamiento contemporáneo. Buenos Aires: Paidós - ICE $-\mathrm{UAB}$.

Glaser, B. y Strauss, A. (1967). The discovery of grounded theory: strategies for qualitative research. Nueva York: Aldine Publishing Company.

Gutiérrez, A. B. (2005). Las prácticas sociales: Una introducción a Pierre Bourdieu. Córdoba: Ferreyra Editor.

Leborgne, D. Lipietz, A. (1994). Flexibilidad ofensiva, flexibilidad defensiva. Dos estrategias sociales en la producción de los nuevos espacios económicos. En G. Benko y A. Lipietz (Comp.), Las regiones que ganan. Distritos y redes. Los nuevos paradigmas de la geografía económica (pp. 331-361). Valencia: Edicions Alfons el Magnanim Generalitat Valenciana.

Lipietz, A. (1988). Miragens e milagres. Problemas da industrialização no Terceiro Mundo. San Pablo: Nobel.

Lipietz, A. (1994). El posfordismo y sus espacios. Las relaciones capital-trabajo en el mundo. Serie Seminarios Intensivos de Investigación. Documento de trabajo No. 4. Buenos Aires: PIETTE-CONICET.

Lipietz, A. Leborgne, D. (1994). El posfordismo y su espacio. Realidad Económica 122, 117-143.

Magariños de Morentín, J. Á. (1996): Fundamentos lógicos de la semiótica y su práctica. Buenos Aires: Edicial.

Magariños de Morentín, J.Á. (1998). Manual operativo para la utilización de la semiótica de enunciados. Signa. Revista de la Asociación Española de Semiótica, 7, 233-253. Recuperado de http://www.biblioteca.org.ar/libros/15599 $0 . \mathrm{pdf}$

Magariños de Morentín, J. Á. et al. (1997). Esbozo semiótico para una metodología de base en Ciencias Sociales. En Los fundamentos lógicos de la semiótica y su práctica (pp. 247-300). Buenos Aires: Edicial. Hay versión electrónica en http://www.centro-de-semiotica.com.ar/EsbozoSemiotico.html

Martínez Nogueira, R. (2004). Historia, aprendizaje y gestión pública: las políticas dirigidas a la pobreza en el Estado remanente. En J. Bertranou, J. M. Palacio y G. M. Serrano (Comps.) En el pais de no me acuerdo. (Des)memoria institucional e historia de la política social en la Argentina (pp. 145-165). Buenos Aires: Prometeo.

Miller, P. y Rose E., N. (1990). Governing economic life. Economy \& Society, 19(1), 1-31.

Miller, P. y Rose, N. (1988). The Tavistock Programme: The Government of Subjectivity and Social Life. Sociology, 22(2), 171-192.

Neffa, J. C. (2008). IV. El estudio del mercado de trabajo desde la teoría de la regulación. En Teorías económicas del mercado de trabajo. III. Análisis institucionalistas (pp. 207-305.). Buenos Aires: FCE.

O’Malley, P. y Palmer, D. (1996). Post Keynesian policing. Economy \& Society, 25(2), 137. 
Oszlak, O.y O 'Donnel, G. (1995). Estado y políticas estatales en América Latina: hacia una estrategia de investigación. REDES (Revista de Estudios Sociales de la Ciencia), 2(4), 97-128.

Pike, K. L. (1954). Language in relation to a unified theory of the structure of human behavior. Preliminary Edition. Volumen 1. Glendale: Calif. Summer Institute of Linguistic.

Rice, G. E. (1980). On Cultural Schemata. American Ethnologist, 7(1), 152-171.

Richards, T. y Richards, L. (1995). Using Hierarchical Categories in Qualitative Data Analysis (training report). En: Kelle, U. (Ed.) Computers and Qualitative Methodology. Londres: Sage Publications.

Rigby, D. K. (2011). Management tools and trends 2011: An Executive's Guide. Bain \& Company. Recuperado de h ttp://www.bain.com/publications/articles/Management-tools-trends-2011.aspx

Rodríguez, P. G. (2013). Las concepciones del desarrollo en los programas de promoción de emprendimientos productivos del Ministerio de Desarrollo Humano de la Provincia de Buenos Aires (1990-2007) (tesis doctoral, Universidad Nacional de La Plata). Recuperada de http://www.memoria.fahce.unlp.edu.ar/library?a=d\&c=tesis\&d=Jte832

Rodríguez, P. G. (2014). Los modelos de intervención en un enfoque cognitivo para el análisis de las políticas públicas. En XI Congreso Argentino de Antropología Social (p. 20). Rosario, 23 al 26/7/2014.

Rose, N. (1992). Governing the enterprising self. En Heelas, P. y Morris, P. (Eds.), The values of the enterprise culture: the moral debate (pp. 141-163). Londres: Routledge.

Rose, N. (1996). The death of the social? Re-figuring the territory of government. Economy \& Society, 25(3), 327. (Hay versión en castellano: Rose, 2007).

Rose, N. (2003). Identidad, genealogía, historia. En S. Hall y P. Du Gay (Comps.), Cuestiones de Identidad Cultural (pp. 251-280). España: Amorrortu.

Rose, N. (2007). ¿La muerte de lo social?: Re-configuración del territorio de gobierno. Revista argentina de sociología, 5(8), 113-152.

Rose, N. y Miller, P. (1992). Political Power beyond the State: Problematics of Government. The British Journal of Sociology, 43(2), 173-205.

Rose, N., O'Malley, P. y Valverde, M. (2006). Governmentality. Annual Review of Law and Social Science, 2(1), 83104.

Sabatier, P. A. (1998). An advocacy coalition framework of policy change and the role of policy-oriented learning therein. Policy Sciences, 21(2-4), 129-168.

Van Dijk, T. A. (1996a): Discourse, power and access. En C. Caldas-Coulthard and M. Coulthard (Eds.) Texts and practices. Readings in Critical Discourse Analysis. Londres: Routledge.

Van Dijk, T. A. (1996b). Análisis del discurso ideológico. Versión. Estudios de comunicación y política, 6, 15-43.

Van Dijk, Teun. A. (2000): Ideologia. Una aproximación multidisciplinaria. Barcelona: Gedisa.

Vasilachis de Gialdino, I. (1992). Métodos cualitativos I. Los problemas teórico - epistemológicos. Buenos Aires: CEAL Colección Fundamentos de las ciencias del hombre.

Wilkis, A. (2004). Apuntes sobre la noción de estrategia en Pierre Bourdieu. Revista argentina de sociología, 3, 118-130.

\section{Notas}

1 Copyright de Qualitative Solutions and Research, Sidney, Australia, 2008. Al comienzo de la investigación se utilizó la versión 8. En el transcurso de ella se actualizó a la versión 9 y posteriormente a la 10, a medida que fueron lanzadas al mercado.

2 Charaudeau y Maingueneau (2005, 337) definen la intertextualidad como "el conjunto de las relaciones explícitas o implícitas que un texto o un grupo de textos determinado mantiene con otros textos". Ejemplos de relaciones explícitas son la cita, la paráfrasis, el comentario y la exégesis.

3 Para la noción de "memoria institucional” véase Bertranou (2004) y Martínez Nogueira (2004).

4 La Gestión Duhalde va desde 1992 hasta 1999 y corresponde a la gestión de Hilda González de Duhalde al frente del organismo como Presidenta Honoraria del Consejo Provincial de la Mujer (CPM) y luego del Consejo Provincial de la Familia y Desarrollo Humano (CPFyDH). La Gestión Ruckauf abarca los años 2000 y 2001 y corresponde a la 
Presidencia Honoraria de María Isabel Zapatero de Ruckauf en el CPFyDH. La Gestión West abarca los años 2002 y 2003, cuando el Lic. Mariano West fue Ministro de Desarrollo Humano y Trabajo. La Gestión MDH incluye los mandatos de los Ministros de Desarrollo Humano Juan Pablo Cafiero y Jorge Varela, entre 2004 y 2007.

5 Los modelos de intervención como dispositivos de gubernamentalidad han sido descritos en un trabajo anterior (Rodríguez, 2014). Para una exposición más detallada véase Rodríguez (2013).

6 El estudio halló que los organismos provinciales cuentan con una variante propia del modelo de intervención hallado en los organismos internacionales y que ésta difiere fundamentalmente en los instrumentos de intervención. Los organismos internacionales focalizan las categorías más abstractas, como los objetivos estratégicos, las estrategias de intervención y, en menor medida, las tecnologías de gestión, y algunos pocos objetos de intervención, como la pobreza y el desempleo. En cambio, los organismos provinciales y sus trabajadores, quienes son los responsables de la implementación de las políticas, elaboran con mayor detalle los instrumentos y las tecnologías, y consideran algunos objetos de intervención adicionales. Mientras los instrumentos cambian con frecuencia, casi como las gestiones de funcionarios provinciales, cuando menos en su denominación, los componentes más abstractos de los modelos de intervención de los organismos provinciales resultan más estables y han sufrido poca variación a lo largo del período considerado. 